\title{
Liver Segment S2 is Consistently Located at the Dorsal Side of S3 But Sometimes at the Right and/or Caudal Side of S3
}

\author{
By
Sadae WAKIGUCHI, Hiroshi YAMAGUCHI, Yoshinobu NAGAOKA, Shintaro MIYAMOTO, Gen MURAKAMI*, Fumitake HATA** and Koichi HIRATA** \\ Undergraduate students of Sapporo Medical University School of Medicine \\ * Department of Anatomy, Sapporo Medical University School of Medicine \\ **First Department of Surgery, Sapporo Medical University School of Medicine \\ - Received for Publication, December 19, 2000 -

\begin{abstract}
Key Words: Liver segment, Lateral sector, Frontal section, Hilar region
Summary: After preparing the frontal section including the origin of the left portal trunk at the hilar region, the left anatomical lobes of 111 human livers were dissected to reveal the segmental configuration based on the supplying portal vein branches. S2 was consistently located dorsal to S3. However, in contrast to the description in common textbooks for medical students, $19.8 \%$ of the specimens carried a paradoxical segmental configuration showing a "caudal and/or rightward" $S 2$ in combination with a "cranial and/or leftward" $S 3$ in the frontal section through the ventral part of the hilar region. The caudal and rightward cases were associated with a specific arrangement of S2 and S3 segmental stems in which the $\mathbf{S 3}$ stem ran relatively upward to spread over $\mathbf{S 2}$ or both stems ran almost horizontal, respectively. In routine diagnostic radiology, identification of $S 2$ and $S 3$ might sometimes be biased by the generally accepted notion that $\mathbf{S 2}$ should be located at the dorsal, cranial and left side of $\mathrm{S3}$.
\end{abstract}

Although numerous investigations have reported the segmental configurations of the human liver, to our knowledge, few anatomical studies have been performed using frontal or coronal sections. However, frontal sections are routinely used in diagnostic radiology, especially in magnetic resonance imaging. We considered that anatomical data on the frontal section of the liver is required not only for the undergraduate medical education but also, possibly, for having clinical relevance. Moreover, in all 3 dimensional representations of the liver in previous reports and textbooks examined by the authors, there were no labels indicating dimensions such as cranial and leftward. This is particular free for cast specimens of the liver (Couinaud, 1989; Gadzijev and Ravnik, 1996). These dimensions are, however, thought to be consistently identifinable without any labels.

We had believed that Couinaud's liver segment S2 (S3) is located at the dorsal (ventral), left (right) and cranial (caudal) sides of S3 (S2) in the left anatomical lobe. In textbooks, however, there are some differences in which of the 3 dimensions is adopted; for example, in the general classification of the primary liver cancer in Japan (Liver Cancer Study Group of Japan, 1997), "dorsal or ventral" is used as the most essential criteria, whereas, in general textbooks of diagnostic ultrasound (Japanese Medical Association, 1991) or surgery (Muto K, Tanabe T, 1995) for undergraduate medical students, an explanation using "upper or lower" (i.e., cranial-caudal axis) is adopted. Therefore, we speculated that most of the students recognize S2 as the upper portion of the left anatomical lobe and S3 as the lower portion. Also in the most widely used textbook of anatomy in English (Williams, 1995) the terms "upper and lower" are commonly used for topographical descriptions. However, a famous German textbook (Loeweneck and Feifel, 1993) employs two of the 3 dimensions (i.e., the "dorsolateral" and "ventromedial") for topographical characterization of S2 and S3. 
Consequently, the aim of this study was to examine the segmental configuration of S2 and S3 in the frontal section. We selected a specific frontal plane running through the origin of the left portal trunk because this plane is easy to reproduce not only in many cadaveric specimens but also in diagnostic radiology.

\section{Materials and Methods}

One hundred and eleven liver specimens were obtained from formaline-fixed cadavers that had been donated to Sapporo Medical University, Health Science University of Hokkaido School of Dentistry, Hokkaido University School of Medicine or Tohoku University School of Medicine for medical and dental education and research. Liver specimens with macroscopic tumors or severe cirrhosis were not included in the study sample.

To correctly identify the 3 dimensions (i.e., the cranio-caudal, left-right and ventral-dorsal axes), three thin bamboo sticks were inserted into the liver before its removal from the cadaver. Using a large brain knife, we then cut the liver into the dorsal and ventral halves along a frontal plane including the origin of the left portal trunk, paying a special attention to the actual positon of the liver in the cadaver. Our cut plane ran through the ventral portion of the hilar region and it consistently included the left anatomical lobe. After cutting, we made a Xerox copy of both cut surfaces. Then, we carefully dissected and identified the segmental portal branches not only from the cut surfaces but also from the visceral (caudal) surface of the liver. Finally, the topographical arrangment of the segments was drawn onto the Xerox copy.

The left surgical lobe was dissected in all 111 specimens to identify "paradoxical" configurations of S2 and S3, such as S2 rightward of and caudal to S3. In 86 of the 111 specimens, detailed dissection and depiction were performed over the entire cut surface to reveal the location of any segmental borders in the frontal section. The segments were identified according to portal vein ramification. Livers in which identification was difficult due to the complex pattern of ramification were not included in the study sample.

\section{Results}

Paradoxical arrangements of S2 and S3 were found in the frontal section of $19.8 \%$ ( 22 of 111 specimens); cranial S3 in $10.8 \%$ (12) and leftward $\mathrm{S} 3$ in $9.0 \%(10)$. Some livers, especially those with a leftward S3 (see below), showed both features, but we classified them into either of the above categories in the present study.

In livers with a cranial S3 (12), this segment is usually adjacent to $\mathrm{S} 4$ on the cut surface, but there were exceptions in which S3 formed an island facing the cranial diaphragmatic surface and was surrounded by $S 2$ from the caudal side in the frontal section (Fig. 1A). Livers with a cranial S3 were associated with a specific arrangement of S2 and S3 segmental stems in which the $\$ 3$ stem ran relatively upward and divided into subsegmental branches. Because those branches were distributed along the curved, cranial diaphragmatic surface, they tended to spread over $\mathrm{S} 2$ in the dorsal part of the left anatomical lobe (Fig. 1B). The left hepatc vein (LHV) was usually cut at the border between S2 and S3. The segmental branches were cut along the frontal section relatively peripheral to their course (i.e., at subsegmental levels).

In livers with a lateral S3 (10), however, S3 occupied almost all of the left half of the cut surface in the left anatomical lobe. The LHV usually ran along the segmental border in the cut surface. Due to the oblique course of the LHV from the inferolateral side (the caudal and left side) to the superomedial side (cranial and right side), S3 tended to be located not only leftward of but also cranial to S2 (Fig. 2). In all 10 livers, both the S2 and S3 segmental stems were clearly identified and, notably,

\section{Explanation of Figures}

\section{Plate I}

Fig. 1. Anatomical representation of a cranial S3 in the frontal section.

A (B) is the dorsal (ventral) half of the specimen after cutting along the frontal plane along the origin of the left portal trunk ( $\mathrm{L}$, cut). A shows the cut surface including segmental borders in black dotted lines, while in B, the complementary cut surface (surrounded by a white dotted line) as well as the upper diaphragmatic surface were partly dissected to show the intrahepatic vessels. Note that the segmental portal branch to $\mathrm{S} 3$ and its subdivisions spread along the convex-shaped, upper surface and hang over S2. II (2), III (3), IV (4) and VIII, liver segments (according to segmental portal vein); LHV or MHV, left or middle hepatic vein; M, main portal trunk; SP, Spiegel's lobe. 

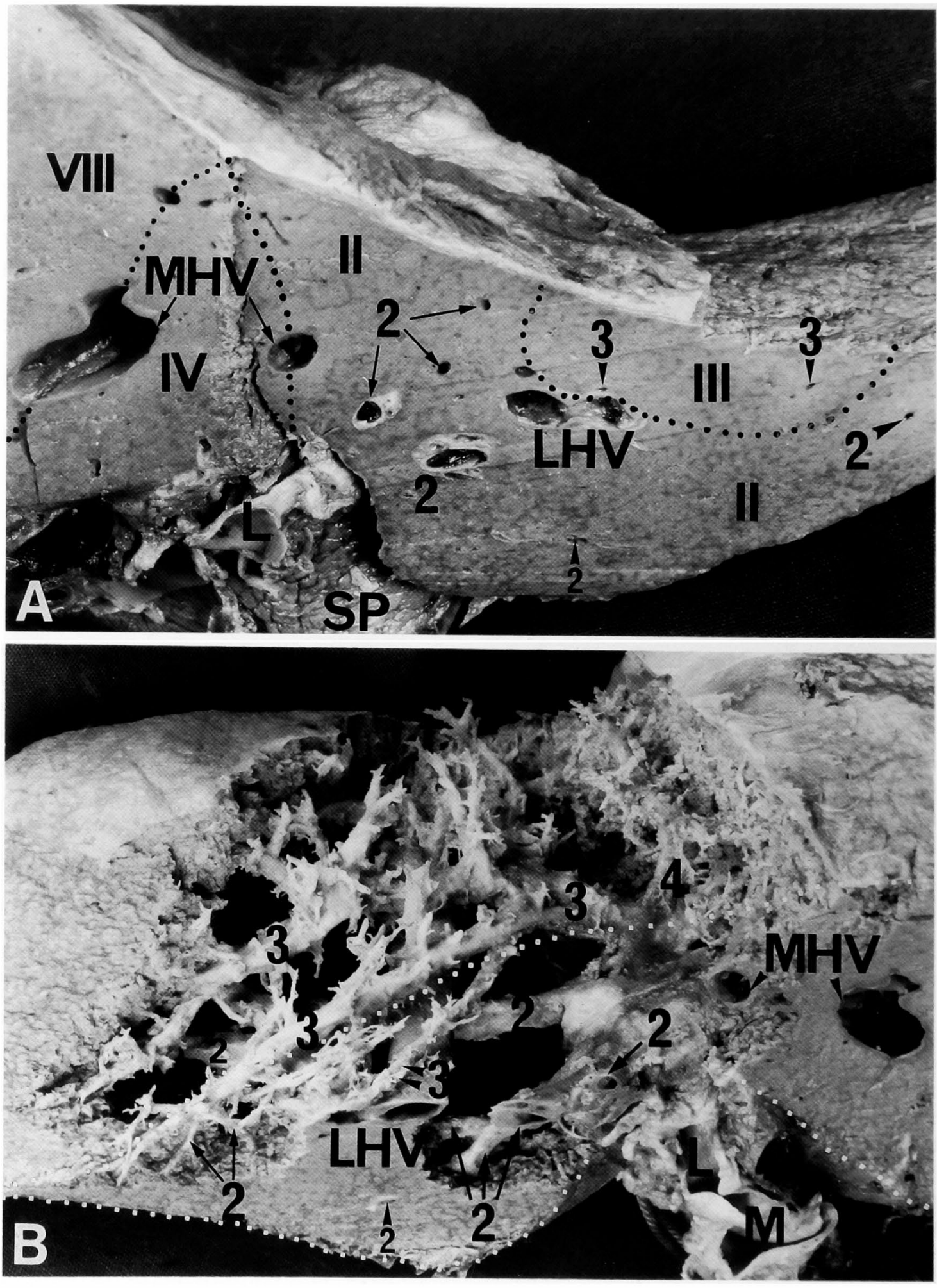
they ran almost horizontal dorsally ( $\mathrm{S} 2$ stem) or ventrally ( 33 stem) to the LHV. The S2 stem was cut along the frontal section. Thus, most of S2 was included in the dorsal half.

In 86 of the 111 livers, we were able to depict the entire segmental configuration in the left anatomical lobe, including paradoxical, cranial or leftward, S3 configurations (see above). In the frontal section, S2 occupied all of the left anatomical lobe in $64.0 \%$ (55 of these 86 specimens). In contrast, S3 occupied all of the lobe in only $2.3 \%$ ( 2 of livers). Notably, in the frontal section through the ventral part of the hilar region, generally-accepted configurations were observed in only $15.1 \%$ (13 of livers); cranial S2 and caudal S3 (4), leftward S2 and rightward S3 (none) or a combination of configurations; i.e., cranial and leftward $S 2$ and caudal and rightward S3 (9). Therefore, S2 was usually located adjacent to as well as dorsal to the ventral portion of the hilar region, whereas S3 occupied a more ventral portion of the liver than generally expected.

\section{Discussion}

The present study was concerned with the anatomy on a specific frontal section of the liver due to its high reproducebility. Accordingly, we will not discuss the general segmental configurations in the left anatomical lobe (that is being prepared for publication in article). Moreover, the specific frontal section seems not to be usually adopted in routine clinical examinations. Nevertheless, the frontal section through the origin of the left portal trunk seems to be convenient plane for comparison with other frontal sections because the position is easily identified in diagnostic radiology. The present results revealed that the "paradoxical" configuration of S2 and S3, such as a rightward and caudal S2, is not a rare morphology in a specific plane but an usual variation. Because previous anatomical studies often ignored the dimensions of specimens or they hypothesized that the dimensions are fundamentally definable (see Introduction), it is difficult to evaluate the real topographical relations between liver segments even in detailed cast-based descriptions such as those used in atlases of intrahepatic vessels (Couinaud, 1989; Gadzijev and Ravnik, 1996). In particular, the rotation of the liver along the craniocaudal axis, if occurring during cast making, seems not to be recognized. Such a rotation, even if it is slight, is likely to affect the topographical relations at the border area between the segments. However, surgeons might not require such detailed information on the 3-dimensional topography of segments, because of the flexible shape and position of the liver during surgery. Nevertheless, for medical education as well as diagnostic radiology and ultrasound, basic 3-dimensional topography is critical.

Our results revealed that S2 is consistently located dorsal to S3 even when paradoxical observations such as lower S2 is combined in the frontal section. Therefore, the terminology used in the general classification of primary liver cancer (Liver Cancer Study Group of Japan, 1997) is correct, although S2 is located cranial rather than dorsal to S3 in its schematic representation. We suspect that, in routine diagnostic radiology, identification of S2 and S3 is biased by the generally accepted notion that $\mathrm{S} 2$ should be located at the dorsal, cranial and left side of S3.

\section{Acknowledgments}

We are grateful to Professor Y Dodo (Tohoku University School of Medicine), Professor M Watanabe (Hokkaido University School of Medicine) and Professor T Yajima (Health Science University of Hokkaido) for permission to use their materials.

\section{References}

1) Couinaud C. Surgical anatomy of the liver revisited. Couinaud, Paris, 1989.

2) Gadzijev E and Ravnik D. Atlas of applied internal liver anatomy. Springer, Wien, 1996.

3) Liver Cancer Study Group of Japan. Classification of primary liver cancer. First English edition. Kanehara, Tokyo, pp 2-3, 1997.

4) Japanese Medical Association. $\mathrm{ABC}$ in the abdominal ultrasound (Fukubu-Echo no $\mathrm{ABC}$ ) (in Japanese). IgakuShoin, Tokyo, pp 138-143, 1991.

5) Muto $K$ and Tanabe T. Standard Surgery (Hyojun Gekagaku) (in Japanese), 7th ed, Igaku-Shoi, Tokyo, pp 603-622, 1995.

6) Williams PL. Gray's Anatomy, 38th ed. Churchill Livingstone, London, pp 1797-1799, 1995.

7) Loeweneck $\mathrm{H}$ and Feifel $\mathrm{G}$. Bauch, In: von Begründet and Lanz T (eds), Plaktische Anatomie. Band 2, Teil 6, Springer, Berlin, pp 227-231, 1993. 

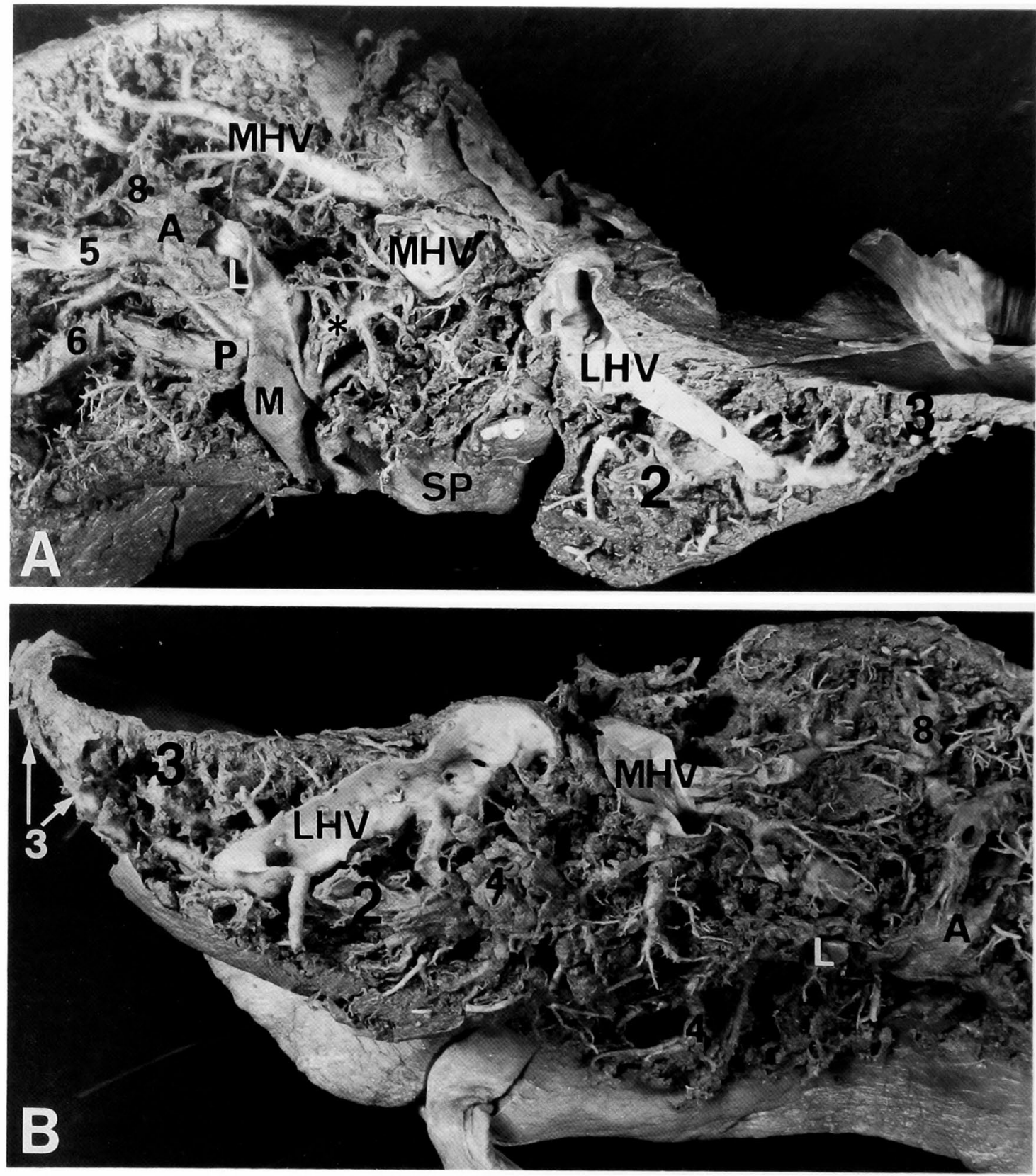

\section{Plate II}

Fig. 2. Anatomical representation of a leftward $\mathrm{S} 3$ in the frontal section.

A (B) is the dorsal (ventral) half of the specimen. Segmental portal branches were dissected from the cut surface as well as from the visceral (caudal) surface in both halves. The hilar trifurcation is evident; i.e., the main portal trunk (M) divides into the left trunk (L, cut) and the anterior (A) and posterior (P) sectorial trunks. A thick, paracaval caudate branch (asterisk) originates from $\mathrm{M}$, crosses behind $\mathrm{L}$ and supplies an area immediately dorsal to MHV. The segmental portal branches to S2 and S3 (2 and 3) run almost horizontal to appear in the cut surface at the left (3) or right (2) side of the LHV. 4, 5, 6 and 8, segmentalportal branches; SP, Spiegel's lobe; LHV or MHV, left or middle hepatic vein. 